\title{
SIGNATURES OF LINKS AND FINITE TYPE INVARIANTS OF CYCLIC BRANCHED COVERS
}

\author{
STAVROS GAROUFALIDIS \\ Dedicated to Mel Rothenberg.
}

\begin{abstract}
Recently, Mullins calculated the Casson-Walker invariant of the 2-fold cyclic branched cover of an oriented link in $S^{3}$ in terms of its Jones polynomial and its signature, under the assumption that the 2 -fold branched cover is a rational homology 3 -sphere. Using elementary principles, we provide a similar calculation for the general case. In addition, we calculate the LMO invariant of the $p$-fold branched cover of twisted knots in $S^{3}$ in terms of the Kontsevich integral of the knot.
\end{abstract}

\section{Contents}

1. Introduction 1

2. A reduction of Theorem $1 \quad 3$

3. Some linear algebra 4

4. Proof of Theorem 1

4.1. The Casson-Walker-Lescop invariant of 3-manifolds 5

4.2. A construction of 2-fold branched covers 6

4.3. Proof of Claim 2.1

5. Proof of Theorem $2 \quad 8$

$\begin{array}{lr}\text { References } & 10\end{array}$

\section{IntRoduction}

Given an oriented link $L$ in (oriented) $S^{3}$, one can associate to it a family of (oriented) 3-manifolds, namely its $p$-fold cyclic branched covers $\Sigma_{L}^{p}$, where $p$ is a positive integer. Using these 3-manifolds, one can associate a family of integer-valued invariants of the link $L$, namely its $p$-signatures, $\sigma_{p}(L)$. These signatures, being concordance invariants, play a key role in the approach to link theory via surgery theory.

On the other hand, any numerical invariant of 3-manifolds, evaluated at the p-fold branched cover, gives numerical invariants of oriented links. The seminal ideas of mathematical physics, initiated by Witten [Wi] have recently produced two axiomatizations (and constructions) of numerical invariants of links and 3-manifolds; one under the name of topological

Date: This edition: September 1, 1998; First edition: November 10, 1997.

The author was partially supported by an NSF grant DMS-98-00703.

This and related preprints can also be obtained at http://www. math.brown.edu/ stavrosg 1991 Mathematics Classification. Primary 57N10. Secondary 57M25.

Key words and phrases: Casson-Walker Lescop invariant, cyclic branched covers, signatures of links, finite type invariants, LMO invariant. 
quantum field theory (e.g. [At, RT1, RT2]) and another under the name of finite type invariants (e.g. [Oh, LMO, BGRT]). Moreover, each of these two approaches offers a conceptual unification of previously known numerical invariants of links and 3-manifolds, such as the Casson invariant $[\mathrm{AM}]$ and the Jones polynomial $[\mathrm{J}]$.

It turns out that the Casson invariant $\lambda$, extended to all rational homology 3-spheres by Walker [Wa], and further extended to all 3-manifolds by Lescop [Le] equals (up to a sign) to twice the degree 1 part of a graph-valued invariant of 3-manifolds [LMO, LMMO] which turns out to be a universal finite type invariant of integral homology 3-spheres, [L].

Recently D. Mullins [Mu] discovered a beautiful relation between the value of CassonWalker invariant of the 2-fold branched cover of a link $L$ in $S^{3}$ in terms of the link's (2)signature $\sigma(L)=\sigma_{2}(L)$ and the value of its Jones polynomial $J_{L}$ at -1 , under the assumption that the 2 -fold branched cover is a rational homology 3 -sphere. It is a natural question to ask whether this assumption is really needed. We can now state our result (where the nullity $\nu(L)$ of a link $L$ can be defined as the first betti number of $\Sigma_{L}^{2}$ ):

Theorem 1. For an oriented link $L$ in $S^{3}$ we have:

$$
i^{\sigma(L)+\nu(L)} \lambda\left(\Sigma_{L}^{2}\right)=\frac{1}{6} J_{L}^{\prime}(-1)+\frac{1}{4} J_{L}(-1) \sigma(L)
$$

A few remarks are in order:

Remark 1.1. In case $\Sigma_{L}^{2}$ is a rational homology 3-sphere, the above formula is Mullin's theorem, as expected. See also Remark 2.2.

Remark 1.2. For the class of links such that $\Sigma_{L}^{2}$ is a rational homology 3-sphere (e.g. for all knots), there is a skein theory relation of the signature, see [Li, Mu]. However, the literature on signatures seems to be avoiding the rest of the links. Our result shows that such a restriction is not necessary.

Remark 1.3. Mullin's proof uses the Kauffman bracket definition of the Jones polynomial $[\mathrm{Ka}]$, and oriented, as well as unoriented, smoothings of the link that are special to the Jones polynomial. Our proof, simpler and derived from first principles, does not use any of these special properties of the Jones polynomial. In addition, it provides a hint for general relations between link signatures and finite type link and 3-manifold invariants.

Corollary 1.4. If $L$ is a link with nullity at least 4 , then $J_{L}(-1)=J_{L}^{\prime}(-1)=0$.

It is natural to ask whether Theorem 1 can be extended to the case of more general covers (such as $p$-fold branched covers), as well as the case of more general 3-manifold invariants, such as the LMO invariant $Z^{L M O}$, or its degree at most $n$ part $Z_{n}^{L M O}$. In this direction, we have the following partial result. Let $D_{m} K$ denote the $m$-fold twisted double of a knot $K$ in $S^{3}$, see Figure 1.

Theorem 2. Fix a knot $K$ in $S^{3}$ and integers $p, m, n$. Then $Z_{n}^{L M O}\left(\Sigma_{D_{m} K}^{p}\right)$ depends only on $p, m$ and the degree $2 n$ part of the Kontsevich integral [Ko] of $K$.

With the above notation, setting $n=1$ we obtain that:

\section{Corollary 1.5.}

$$
\lambda\left(\sum_{D_{m} K}^{p}\right)=a_{p, m} \Delta^{\prime \prime}(K)(1)+b_{p, m}
$$

where $\Delta(K)$ is the Alexander-Conway polynomial of $K,[\mathrm{C}, \mathrm{Ka}]$ and $a_{p, m}, b_{p, m}$ are constants depending on $p, m$. 


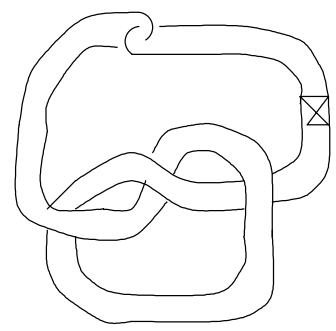

Figure 1. The $m$-twisted double of a trefoil. In the region marked by $X$ are $m-3$ full twists.

The above corollary was obtained independently by Ishibe [I] for general $m, p$, Hoste [Ho] for $m=0$ and Davidow [Da] for $m=-1, p \equiv \pm 1 \bmod 6$.

We wish to thank Daniel Ruberman for numerous encouraging, enlightening and clarifying conversations.

\section{A Reduction of Theorem 1}

Before we get involved in calculations, we should mention that the proof of Theorem 1 is an application of skein theory and the following two properties, together with their philosophical proof:

P1 The Casson-Walker-Lescop invariant satisfies a 3-term relation. This holds since the Casson-Walker-Lescop invariant is a finite type 3 invariant (at least restricted to the set of rational homology 3 -spheres, [GO]).

P2 A crossing change or a smoothening of a link $L$, results in (two) surgeries along the same knot in $S^{3}$. This holds since the 2-fold branched cover of a disk $D^{2}$ (branched along two points) is an annulus, thus the 2-fold branched cover of $D^{2} \times I$ branched along two arcs is a solid torus $T$.

All links and 3-manifolds in this paper are oriented. With an eye in equation (1), we define for a link $L$ in $S^{3}$,

$$
\alpha(L) \stackrel{\text { def }}{=} 1 / 6 J_{L}^{\prime}(-1), \quad \beta(L) \stackrel{\text { def }}{=} i^{\sigma(L)+\nu(L)} \lambda\left(\Sigma_{L}^{2}\right), \quad \text { and } \quad \gamma(L) \stackrel{\text { def }}{=} 1 / 4 J_{L}(-1) \sigma(L) .
$$

A triple of links $\left(L^{+}, L^{-}, L^{0}\right)$ is called bordered if there is an embedded disk $D^{3}$ in $S^{3}$ that locally intersects them as in figure 2 .

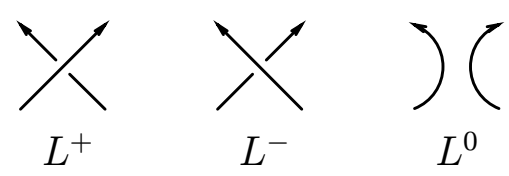

Figure 2. A bordered triple of links $\left(L^{+}, L^{-}, L^{0}\right)$.

For a bordered triple $\left(L^{+}, L^{-}, L^{0}\right)$, the skein relation $t J_{L^{+}}(t)-t^{-1} J_{L^{-}}(t)=\left(t^{1 / 2}-t^{-1 / 2}\right) J_{L^{0}}(t)$ of the Jones polynomial implies that:

$$
\alpha\left(L^{+}\right)-\alpha\left(L^{-}\right)=-2 i \alpha\left(L^{0}\right)+\frac{J_{L^{+}}(-1)}{6}+\frac{J_{L^{-}}(-1)}{6}
$$

Thus, the following claim: 
Claim 2.1. For a bordered triple $\left(L^{+}, L^{-}, L^{0}\right)$, we have:

$$
\beta\left(L^{+}\right)-\beta\left(L^{-}\right)+2 i \beta\left(L^{0}\right)=\gamma\left(L^{+}\right)-\gamma\left(L^{-}\right)+2 i \gamma\left(L^{0}\right)+\frac{J_{L^{+}}(-1)}{6}+\frac{J_{L^{-}}(-1)}{6}
$$

together with the initial condition $\alpha$ (unknot $)=\beta$ (unknot $)-\gamma($ unknot $)=0$, proves Theorem 1. The rest of the paper is devoted to the proof of the above claim.

Remark 2.2. Jones and Mullins use a similar but different skein theory for the Jones polynomial, namely $t^{-1} V_{L^{+}}(t)-t V_{L^{-}}(t)=\left(t^{1 / 2}-t^{-1 / 2}\right) V_{L^{0}}(t)$. The polynomials $V_{L}$ and $J_{L}$ are easily seen to be related by: $J_{L}(t)=(-1)^{|L|-1} V_{L}\left(t^{-1}\right)$, where $|L|$ is the number of components of $L$. With our choice, it turns out that $J_{L}(1)=2^{|L|-1}$, a positive integer, which is natural from the point of view of quantum groups and perturbative Chern-Simons theory. Furthermore, Mullins is evaluating $V_{L}$ at -1 with the the rather nonstandard convention that $\sqrt{-1}=-i$, whereas we are evaluating $J_{L}$ at -1 with the convention that $\sqrt{-1}=i$.

\section{Some Linear Algebra}

We begin by reviewing three important invariants of symmetric matrices $A$. All matrices considered have real entries, and $B^{T}$ denotes the transpose of the matrix $B$. Two matrices $B$ and $B^{\prime}$ are called similar if $B^{\prime}=P B P^{T}$, for a nonsingular matrix $P$. Given a symmetric matrix $A$, we denote by $\nu(A), \sigma(A)$ its nullity and signature respectively. A lesser known invariant, the sign $\operatorname{sgn}^{\prime}(A)$ of $A$, can be obtained as follows: bring $A$ to the form $P A P^{T}=$ $\left(\begin{array}{cc}A^{\prime} & 0 \\ 0 & 0\end{array}\right)$ where $A^{\prime}, P$ are nonsingular, [Ky]. Then, we can $\operatorname{define} \operatorname{sgn}^{\prime}(A)=\operatorname{sgn}^{\prime}\left(\operatorname{det}\left(A^{\prime}\right)\right)$, with the understanding that the sign of the determinant of a $0 \times 0$ matrix is 1 . It is easy to see that the result is independent of $P$; moreover, it coincides with Lescop's definition [Le, Section 1.3]. Notice that the signature, nullity and sign of a matrix do not change under similarity transformations.

We call a triple of symmetric matrices $\left(A_{+}, A_{-}, A_{0}\right)$ bordered if

$$
A_{+}=\left(\begin{array}{cc}
a & \rho \\
\rho^{T} & A_{0}
\end{array}\right) \text { and } A_{-}=\left(\begin{array}{cc}
a+2 & \rho \\
\rho^{T} & A_{0}
\end{array}\right)
$$

for a row vector $\rho$. The signatures and nullities of a bordered triple are related as follows, $[\mathrm{C}]$ :

$$
\left|\nu\left(A_{ \pm}\right)-\nu\left(A_{0}\right)\right|+\left|\sigma\left(A_{ \pm}\right)-\sigma\left(A_{0}\right)\right|=1
$$

Thus, in a bordered triple, the nullity determines the signature, up to a sign. A more precise relation is the following:

Lemma 3.1. The sign, nullity and signature in a bordered triple are related as follows:

$$
\begin{aligned}
& \sigma\left(A_{ \pm}\right)-\sigma\left(A_{0}\right)= \begin{cases}0 & \text { if }\left|\nu\left(A_{ \pm}\right)-\nu\left(A_{0}\right)\right|=1 \\
\operatorname{sgn}^{\prime}\left(A_{ \pm}\right) \operatorname{sgn}^{\prime}\left(A_{0}\right) & \text { otherwise. }\end{cases} \\
& \nu\left(A_{ \pm}\right)-\nu\left(A_{0}\right)= \begin{cases}0 & \text { if }\left|\sigma\left(A_{ \pm}\right)-\sigma\left(A_{0}\right)\right|=1 \\
\operatorname{sgn}^{\prime}\left(A_{ \pm}\right) \operatorname{sgn}^{\prime}\left(A_{0}\right) & \text { otherwise. }\end{cases}
\end{aligned}
$$

Moreover, if $\epsilon_{x} \stackrel{\text { def }}{=} \operatorname{sgn}^{\prime}\left(A_{x}\right) i^{\sigma\left(A_{x}\right)+\nu\left(A_{x}\right)}$ for $x \in\{+,-, 0\}$, then we have:

$$
\epsilon_{+}=\epsilon_{-}=i \epsilon_{0} \text {. }
$$


Proof. By similarity transformations, we can assume that:

$$
A_{+}=\left(\begin{array}{cc}
a & \rho \\
\rho^{T} & 0
\end{array}\right) \oplus D, A_{+}=\left(\begin{array}{cc}
a+2 & \rho \\
\rho^{T} & 0
\end{array}\right) \oplus D, A_{0}=D \oplus[0]^{r}
$$

where $D$ is a nonsingular, diagonal matrix, $[0]^{r}$ is the zero $r \times r$ matrix, $\rho$ is a $1 \times r$ vector and $a$ a real number. Since the nullity, signature and sign of the matrix $\left(\begin{array}{cc}a & \rho \\ \rho^{T} & 0\end{array}\right)$ are given by:

\begin{tabular}{|l|c|c|c|}
\hline & $\rho=a=0$ & $\rho=0, a \neq 0$ & $\rho \neq 0$ \\
\hline nullity & $r+1$ & $r$ & $r-1$ \\
\hline signature & 0 & $\operatorname{sgn}^{\prime}(a)$ & 0 \\
\hline sign & 1 & $\operatorname{sgn}^{\prime}(a)$ & -1 \\
\hline
\end{tabular}

the result follows by a case-by-case argument.

Remark 3.2. For future reference, we mention that the determinants of a bordered triple of matrices are related by:

$$
\operatorname{det}\left(A_{+}\right)-\operatorname{det}\left(A_{-}\right)+2 \operatorname{det}\left(A_{0}\right)=0
$$

This follows easily by expanding the first two determinants along the first column.

Given an oriented link $L$ in $S^{3}$, choose of Seifert surface of it, together with a basis for its homology and and consider the associated Seifert matrix $E_{L}$. Recall that the nullity $\nu(L)$, signature $\sigma(L)$ and sign $\operatorname{sgn}^{\prime}\left(E_{L}\right)$ of $L$ are defined as the nullity, signature and sign of the symmetrized Seifert matrix $E_{L}+E_{L}^{T}$. It turns out that the signature and nullity of a link are independent of the Seifert surface chosen, and that $\nu(L)=\beta_{1}\left(\Sigma_{L}^{2}\right)$, where $\beta_{1}$ is the first betti number. On the other hand, $\operatorname{sgn}^{\prime}\left(E_{L}\right)$ depends on the Seifert matrix.

It is easy to see that given a bordered triple $\left(L^{+}, L^{-}, L^{0}\right)$ of links, one can construct a triple of Seifert matrices so that the associated triple of symmetrized Seifert matrices is bordered, $[\mathrm{C}]$.

\section{Proof of Theorem 1}

4.1. The Casson-Walker-Lescop invariant of 3-manifolds. Given an (integrally) framed oriented r-component link $\mathcal{L}$ in $S^{3}$ (with ordered components), let $S_{\mathcal{L}}^{3}$ denote the closed 3manifold obtained by Dehn surgery on $\mathcal{L}$. Its linking matrix, $F(\mathcal{L})$ gives a presentation of $H_{1}\left(S_{\mathcal{L}}^{3}, \mathbb{Z}\right)$. Notice that $\nu(F(\mathcal{L}))=\beta_{1}\left(S_{\mathcal{L}}^{3}\right)$. The Casson-Walker-Lescop invariant $\lambda$ of $S_{\mathcal{L}}^{3}$ is defined by:

$$
\lambda\left(S_{\mathcal{L}}^{3}\right)=\operatorname{sgn}^{\prime}(F(\mathcal{L}))\left(D(\mathcal{L})+H_{0}(\mathcal{L})+H_{1}(\mathcal{L})+H_{2}(\mathcal{L})\right), \text { where }
$$




$$
\begin{aligned}
D(\mathcal{L}) & =\sum_{\emptyset \neq \mathcal{L}^{\prime} \subseteq \mathcal{L}} \operatorname{det}\left(F\left(\mathcal{L} \backslash \mathcal{L}^{\prime}\right)\right) \zeta\left(\mathcal{L}^{\prime}\right), \\
H_{0}(\mathcal{L}) & =\frac{\operatorname{det}(F(\mathcal{L}))}{4} \sigma(F(\mathcal{L})) \\
H_{1}(\mathcal{L}) & =-\frac{1}{6} \sum_{j=1}^{r} \operatorname{det}(F(\mathcal{L} \backslash j)) \\
H_{2}(\mathcal{L}) & =\frac{1}{12} \sum_{\emptyset \neq \mathcal{L}^{\prime} \subseteq \mathcal{L}} \operatorname{det}\left(F\left(\mathcal{L} \backslash \mathcal{L}^{\prime}\right)\right)(-1)^{\left|\mathcal{L}^{\prime}\right|} L_{8}\left(\mathcal{L}^{\prime}\right),
\end{aligned}
$$

$\zeta(\mathcal{L})$ is a special value of (a derivative of) the multivariable Alexander polynomial of $\mathcal{L}$ and $L_{8}(\mathcal{L})$ is a polynomial in the linking numbers $l_{a b}(a, b=1 \ldots r)$ of $\mathcal{L}$ given explicitly by:

$$
L_{8}(\mathcal{L})=\sum_{j=1}^{r} \sum_{\sigma \in \operatorname{Sym}_{r}} l_{i \sigma(1)} l_{\sigma(1) \sigma(2)} \ldots l_{\sigma(r-1) \sigma(r)} l_{\sigma(r) i},
$$

where $\operatorname{Sym}_{r}$ is the symmentric group with $r$ letters.

Notice also that since the links $\mathcal{L}$ that we consider will be integrally framed, the Dedekind sums appearing in [Le, definition 1.4.5] vanish.

4.2. A construction of $\mathbf{2}$-fold branched covers. In this section we review the details of some well known construction of 2 -fold branched covers of links in $S^{3}$. For a general reference, see $[\mathrm{Ka}, \mathrm{AK}]$. Given an (oriented) link $L$ in $S^{3}$, choose a Seifert surface $F_{L}$ of $L$, and a basis of its first homology and let $E_{L}$ be its Seifert matrix. Push a bicolar of $F_{L}$ in the interior of $D^{4}$ (the 4-manifold obtained is still diffeomorphic to $D^{4}$ ), and glue two copies of the obtained 4-manifold along $F_{L}$ according to the pattern of [Ka, p. 281]; let $N_{F_{L}}$ denote the resulting 4-manifold, which is a 2 -fold cover of $D^{4}$ branched along $F_{L}$. Its boundary is $\Sigma_{L}^{2}$, the 2-fold cover of $S^{3}$ branched along $L$. In [AK, section 2], Akbulut-Kirby showed that $N_{F_{L}}$ is a 4-dimensional handlebody (i.e. the result of attaching 2-handles along $D^{4}$ ), and that the intersection form with respect to some basis of these 2-handles is the symmetrized Seifert matrix $E_{L}+E_{L}^{T}$ of $L$. Let $\mathcal{L}$ denote the cores in $S^{3}$ of the 2-handles. Thus, $\mathcal{L}$ is a framed link in $S^{3}$ with linking matrix $E_{L}+E_{L}^{T}$, such that Dehn surgery on $\mathcal{L}$ is $\Sigma_{L}^{2}$. Of course, the link $\mathcal{L}$ depends on the choice of Seifert surface of $L$ as well as on a choice of basis on its homology. Akbulut-Kirby $[\mathrm{AK}]$ describe an algorithm for drawing $\mathcal{L}$ and implement it with beautiful pictures, however we will not need the precise picture of the link $\mathcal{L}$ !

Assume now that $\left(L^{+}, L^{-}, L^{0}\right)$ is a bordered triple with admissible Seifert surfaces. Property $\mathbf{P} 1$ of Section 2 implies that there is a solid torus in $\Sigma_{L^{0}}^{2}$ and three simple curves $\alpha^{+}, \alpha^{-}, \alpha^{0}$ in its boundary so that $\Sigma_{L^{x}}^{2}$ is diffeomorphic to surgery on the solid torus $T$ in $\Sigma_{L^{0}}^{2}$ along $\alpha_{x}$, for $x \in\{+,-, 0\}$. Using the argument of [Mu, page p.429], it follows that there is a choice of a standard symplectic basis $\left\{x_{1}, x_{2}\right\}$ for $H_{1}(\partial T, \mathbb{Z})$ so that $\alpha^{+}=x_{2}, \alpha^{-}=2 x_{1}+x_{2}, \alpha^{0}=x_{1}$. In other words, we have: $\left\langle\alpha^{0}, \alpha^{+}\right\rangle=1$ and $\alpha^{-}=2 \alpha^{0}+\alpha^{+}$, where $\langle\cdot, \cdot\rangle$ is the intersection form.

From the above discussion, it follows that there is a triple of framed oriented links (not necessarily bordered!) $\left(\mathcal{L}^{+}, \mathcal{L}^{-}, \mathcal{L}^{0}\right)$ in $S^{3}$ an oriented knot $K$ and an integer $n$ so that $\Sigma_{L^{x}}^{2}=\Sigma_{\mathcal{L}^{x}}^{3}$ for all $x \in\{+,-, 0\}$, and so that $\mathcal{L}^{+}$(resp. $\left.\mathcal{L}^{-}\right)$is the disjoint union of $\mathcal{L}^{0}$ with the framed knot $(K, n)$ (resp. $(K, n+2))$. Thus, the triple $\left(F\left(\mathcal{L}^{+}\right), F\left(\mathcal{L}^{-}\right), F\left(\mathcal{L}^{0}\right)\right)$ of linking matrices is bordered, with nullity, signature and sign equal to that of the triple $\left(L^{+}, L^{-}, L^{0}\right)$. 
4.3. Proof of Claim 2.1. Let us define $\epsilon_{x}=\operatorname{sgn}^{\prime}\left(\mathcal{L}^{x}\right) i^{\sigma\left(L^{x}\right)+\nu\left(L^{x}\right)}$ for $x \in\{-,+, 0\}$, Since the $\sigma\left(\mathcal{L}^{x}\right)=\sigma\left(L^{x}\right)$, Lemma 3.1 implies that $\epsilon_{ \pm}=i \epsilon_{0}$.

We can now calculate as follows:

$$
\begin{aligned}
\beta\left(L^{+}\right)-\beta\left(L^{-}\right)+2 i \beta\left(L^{0}\right) & =\left\{\epsilon_{+} D\left(\mathcal{L}^{+}\right)-\epsilon_{-} D\left(\mathcal{L}^{-}\right)+2 i \epsilon_{0} D\left(\mathcal{L}^{0}\right)\right\} \\
& +\sum_{k=0}^{2}\left\{\epsilon_{+} H_{k}\left(\mathcal{L}^{+}\right)-\epsilon_{-} H_{k}\left(\mathcal{L}^{-}\right)+2 i \epsilon_{0} H_{k}\left(\mathcal{L}^{0}\right)\right\}
\end{aligned}
$$

We claim that:

$$
\begin{aligned}
\epsilon_{+} D\left(\mathcal{L}^{+}\right)-\epsilon_{-} D\left(\mathcal{L}^{-}\right)+2 i \epsilon_{0} D\left(\mathcal{L}^{0}\right) & =0 \\
\epsilon_{+} H_{0}\left(\mathcal{L}^{+}\right)-\epsilon_{-} H_{0}\left(\mathcal{L}^{-}\right)+2 i \epsilon_{0} H_{0}\left(\mathcal{L}^{0}\right) & =\gamma\left(L^{+}\right)-\gamma\left(L^{-}\right)+2 i \gamma\left(L^{+}\right) \\
\epsilon_{+} H_{1}\left(\mathcal{L}^{+}\right)-\epsilon_{-} H_{1}\left(\mathcal{L}^{-}\right)+2 \epsilon_{0} H_{1}\left(\mathcal{L}^{0}\right) & =0 \\
\epsilon_{+} H_{2}\left(\mathcal{L}^{+}\right)-\epsilon_{-} H_{2}\left(\mathcal{L}^{-}\right)+2 \epsilon_{0} H_{2}\left(\mathcal{L}^{0}\right) & =\frac{J_{L^{+}}(-1)}{6}+\frac{J_{L^{-}}(-1)}{6}
\end{aligned}
$$

Before we show the above equations, we let $\mathcal{L}^{0} \cup K^{ \pm}=\mathcal{L}^{ \pm}$, and $I$ denote an arbitrary nonempty sublink of $\mathcal{L}^{0}$ with complement $I^{\prime} \stackrel{\text { def }}{=} \mathcal{L}^{0} \backslash I$.

Using equation (7), it follows that the left hand side of (9) equals to:

$$
\begin{array}{ll}
\sum_{I}\left\{\epsilon_{+} \operatorname{det}\left(F\left(I^{\prime} \cup K^{+}\right)\right)-\epsilon_{-} \operatorname{det}\left(F\left(I^{\prime} \cup K^{-}\right)\right)+2 i \epsilon_{0} \operatorname{det}\left(F\left(I^{\prime}\right)\right)\right\} \zeta(I)+ \\
\left.\sum_{I}\left\{\epsilon_{+} \operatorname{det}\left(F\left(I^{\prime}\right)\right)-\epsilon_{-} \operatorname{det}\left(F\left(I^{\prime}\right)\right)\right)\right\} \zeta(I \cup K)
\end{array}
$$

Using equation (7), and the fact that $\left.F\left(I \cup K^{+}\right), F\left(I \cup K^{-}\right), F\left(I \cup K^{0}\right)\right)$ is a bordered triple of matrices, it follows by Remark 3.2 that the first and second sum shown above vanishes, thus showing equation (9).

In order to show equation (10), use the fact that for a link $L$ in $S^{3}$ we have:

$$
\left|J_{L}(-1)\right|=\left|H_{1}\left(\Sigma^{2}, \mathbb{Z}\right)\right|=i^{-\sigma(L)-2 \nu(L)} J_{L}(-1)
$$

(compare with $[\mathrm{Mu}$, Theorem 2.4], and with Kauffman [Ka], with the understanding that the order of an infinite group is 0 , and keeping in mind Remark 2.2). Thus,

$$
\epsilon_{x} \operatorname{det}\left(F\left(\mathcal{L}^{x}\right)\right)=i^{\sigma\left(L^{x}\right)+\nu\left(L^{x}\right)}\left|H_{1}\left(\Sigma^{2}, \mathbb{Z}\right)\right|=i^{-\nu\left(L^{x}\right)} J_{L^{x}}(-1)=J_{L^{x}}(-1)
$$

for all $x \in\{+,-, 0\}$ (where the last equality above follows from the fact that if $\nu\left(L^{x}\right) \neq 0$, then $\left.J_{L^{x}}(-1)=0\right)$. Since $\sigma\left(\mathcal{L}^{x}\right)=\sigma\left(L^{x}\right)$ for all $x$, it follows that $\epsilon_{x} H_{0}\left(\mathcal{L}^{x}\right)=\gamma\left(L^{x}\right)$ for all $x$, which proves equation $(10)$.

Equation (11) follows in the same way as equation (9) shown above. 
Using (7) and the definition of $H_{2}$, it follows that the left hand side of equation (12) equals to:

$$
\begin{aligned}
& i \epsilon_{0} \quad\left(H_{2}\left(\mathcal{L}^{+}\right)-H_{2}\left(\mathcal{L}^{0}\right)+2 H_{2}\left(\mathcal{L}^{0}\right)\right) \\
& =\frac{i \epsilon_{0}}{12}\left\{\sum_{I} \operatorname{det}\left(F\left(I^{\prime}\right)\right)(-1)^{|I \cup K|} L_{8}\left(I \cup K^{+}\right)+\sum_{I} \operatorname{det}\left(F\left(I^{\prime} \cup K^{+}\right)\right)(-1)^{|I|} L_{8}(I)\right. \\
& -\sum_{I} \operatorname{det}\left(F\left(I^{\prime}\right)\right)(-1)^{|I \cup K|} L_{8}\left(I \cup K^{-}\right)-\sum_{I} \operatorname{det}\left(F\left(I^{\prime} \cup K^{-}\right)\right)(-1)^{|I|} L_{8}(I) \\
& \left.+2 \sum_{I} \operatorname{det}\left(F\left(I^{\prime}\right)\right)(-1)^{|I|} L_{8}(I)\right\} \\
& =\frac{i \epsilon_{0}}{12}\left\{\sum_{I} \operatorname{det}\left(F\left(I^{\prime}\right)\right)(-1)^{|I|}\left(-L_{8}\left(I \cup K^{+}\right)+L_{8}\left(I \cup K^{+}\right)\right)\right\}
\end{aligned}
$$

It is easy to see that if $\left(l_{a b}^{ \pm}\right)=F\left(\mathcal{L}^{ \pm}\right)$and that $K^{ \pm}$is the first ordered component of $\mathcal{L}^{ \pm}$, then

$$
\begin{aligned}
\left.-L_{8}\left(I \cup K^{+}\right)+L_{8}\left(I \cup K^{+}\right)\right) & =2 \sum_{\sigma^{\prime \prime}} l_{1 \sigma^{\prime \prime}(1)}^{+} l_{\sigma^{\prime \prime}(1) \sigma^{\prime \prime}(2)}^{+} \ldots l_{\sigma^{\prime \prime}(r-1) \sigma^{\prime \prime}(r)}^{+} \\
& +2 \sum_{\sigma^{\prime}} l_{\sigma^{\prime}(1) \sigma^{\prime}(2)}^{-} l_{\sigma^{\prime}(2) \sigma^{\prime}(3)}^{-} \ldots l_{\sigma^{\prime}(r+1) 1}^{-}
\end{aligned}
$$

where the summation is over all $\sigma^{\prime}, \in \operatorname{Sym}_{r+1}\left(\right.$ resp. $\left.\sigma^{\prime \prime}\right)$ such that $\sigma^{\prime}(1)=1$ (resp. $\sigma^{\prime \prime}(r+1)=$ 1). Combined with the above, and with (7), (13), the left hand side of equation (12) equals to

$\frac{i \epsilon_{0}}{6}\left(\operatorname{det}\left(F\left(\mathcal{L}^{+}\right)\right)+\operatorname{det}\left(F\left(\mathcal{L}^{-}\right)\right)=\frac{1}{6}\left(\epsilon_{+} \operatorname{det}\left(F\left(\mathcal{L}^{+}\right)\right)+\epsilon_{-} \operatorname{det}\left(F\left(\mathcal{L}^{-}\right)\right)=\frac{1}{6}\left(V_{L^{+}}(-1)+V_{L-}(-1)\right)\right.\right.$ which concludes the proof of equation (12) and of Claim 2.1.

Corollary 1.4 follows from the fact that the Casson-Walker-Lescop invariant of a manifold with betti number at least 4, vanishes, [Le]. Remark 1.1 follows from Theorem 1 and (13).

Remark 4.1. The forth roots of unity in (7) work out in such a way as to obtain the cancellations in equations (9), (10), (11) and (12). The philosophical reason for the cancelation in equation (9) is property $\mathbf{P} 2$ of Section 2.

\section{Proof of Theorem 2}

Fix a knot $K$ in $S^{3}$ and integers $p, m, n$. Our first goal is to give a Dehn surgery description of the $p$-fold branched cover $\Sigma_{D_{m} K}^{p}$ of the $m$-twisted double $D_{m} K$ of $K$.

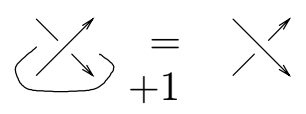

Figure 3. A Dehn twist along a +1 framed unknot intersecting two arcs (in an arbitrary 3-manifold) on the left hand side gives a diffeomorphic 3-manifold with a different embedding of the two arcs.

We begin with a definition: a link $L$ in $S^{3}$ is called $K$-unknotting if it is unit-framed, algebraically split (i.e., one with linking numbers zero and framing \pm 1 on each component), lying in a standard solid torus in $S^{3}$, such that Dehn surgery $S_{L}^{3}$ on $L$ is diffeomorphic to $S^{3}$ 
and such that the image of a meridian of the solid torus in $S_{L}^{3}$ is isotopic to the knot $K$ in $S^{3}$. A $K$-uknotting link $L$ can be obtained by projecting $K$ to a generic plane, choosing a set of crossings that unknot $K$, and placing an unknot with framing \pm 1 around each crossing. The union of these unknots is the desired link $L$, as follows from Figure 3.

We represent a $K$-unknotting link $L$ in the standard solid torus in $S^{3}$ with the left hand side of Figure 3. On the right hand side of the same figure is shown the $m$-twisted double of $K$.
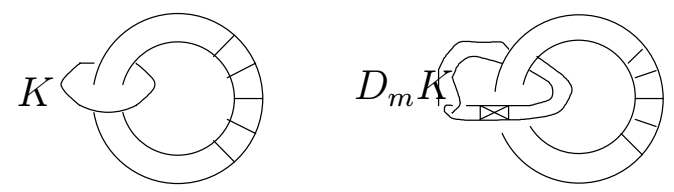

Figure 4. On the left, a $K$-unknotting link $L$. On the right, the $m$-twisted double $D_{m} K$. In the box marked $X$ are $m$ twists.

Next, we construct an $D_{m} K$ uknotting link: using Figure 3, we introduce an extra unitframed unknot $C$ to unknot $D_{m} K$ as shown on the left hand side of Figure 5 , and we isotope the result as shown on the right hand side of the figure. Then, $L \cup C$ is a $D_{m} K$-unknotting link.
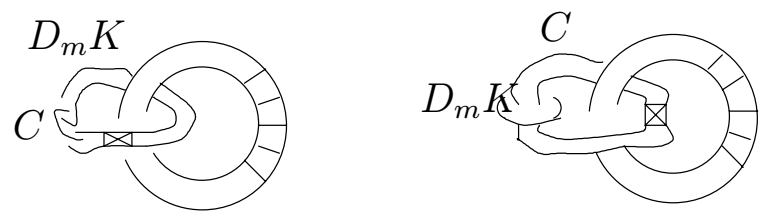

Figure 5. Two isotopic views of $L \cup C$, which is $D_{m} K$-unknotting.

Cutting the meridian disk that $D_{m} K$ bounds on the right hand side of Figure 5, and gluing $p$ of them side by side as in Figure 6, gives a framed link $L(p, m)$ in $S^{3}$, which is a Dehn surgery presentation of $\Sigma_{D_{m} K}^{p}$.

Next, we review some well-known facts about the LMO invariant, and its cousin, the Aarhus integral, [BGRT]. We will assume some familiarity with the standard definitions of finite type invariants of links and 3-manifolds, [B-N, Ko, LMO, BGRT]. Both the LMO invariant and the Aarhus integral of a 3-manifold obtained by Dehn surgery of a link in $S^{3}$ are defined in terms of the Kontsevich integral of the link. For rational homology 3spheres, the LMO invariant equals, properly normalized, to the Aarhus integral, where the normalization of the factor is the order of the first homology group with integer coefficients. The manifolds in question, namely $\Sigma_{D_{m} K}^{p}$, are rational homology 3-spheres, and the order of the first homology group depends only on $m$ and $p$ (since the first homology is given in terms of the Alexander polynomial of $D_{m} K$, which depends only on $m$, evaluated at $p$ th roots of unity). Thus, it suffices to show that the degree at most $n$ part of the Aarhus integral $Z_{n}^{A}\left(\Sigma_{D_{m} K}^{p}\right)$ of $\Sigma_{D_{m} K}^{p}$ depends on the degree at most $2 n$ part of the Kontsevich integral $Z_{2 n}^{K}(K)$ of $K$.

Since the degree at most $d$ part of the Kontsevich integral is the universal Vassiliev invariant of type $d[\mathrm{Ko}, \mathrm{B}-\mathrm{N}]$, it suffices to show that the knot invariant $K \rightarrow Z_{n}^{A}\left(\Sigma_{D_{m} K}^{p}\right)$ is a Vassiliev invariant of type $2 n$. 


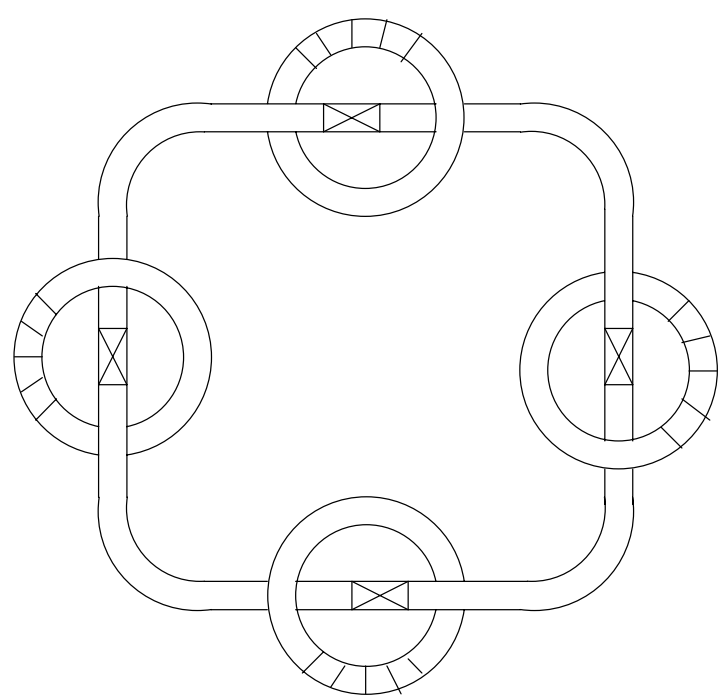

Figure 6. The framed link $L(4, m)$.

A crossing change on $K$ corresponds to an extra component to the $K$-unknotting link $L$ and $p$ extra components to the link $L(p, m)$, thus the alternating sum of $2 n+1$ double points on $K$, corresponds to the alternating sum of a link $L_{\text {alt }} \cup L(p, m)$, where $L_{\text {alt }}$ has $(2 n+1) p$ components, and where we alternate by considering including or not each of the $p$ components. We then consider the Kontsevich integral of $L_{\text {alt }} \cup L(p, m)$, expressed in terms of disjoint union of uni-trivalent graphs, the legs of which we glue according to the definition of the Aarhus integral. Since the linking numbers between the components of $L_{\text {alt }}$ and $L_{\text {alt }} \cup L(p, m)$ are zero, a standard counting argument (compare with [L], [BGRT, part II, Section 4, proof of Theorem 2] and with [GH, proof of Theorem 3])shows that after alernating $2 n+1$ terms, the degree $n$ part of $Z_{n}^{A}\left(L_{\text {alt }} \cup L(p, m)\right)$ vanishes.

Corollary 1.5 follows immediately from Theorem 2, using the fact that the degree 1 part of the LMO invariant equals to the Casson-Walker-Lescop invariant, [LMMO, LMO].

\section{REFERENCES}

[AK] S. Akbulut, R. Kirby, Branched covers of surfaces in 4-manifolds, Math. Ann. 252 (1980) 111-131.

$[\mathrm{AM}] \quad$ S. Akbulut, J. C. McCarthy, Casson's invariant for oriented homology 3-spheres: an exposition, Princeton Math Notes, Princeton, 1990.

[At] M.F. Atiyah, Topological Quantum Field Theories, I.H.E.S. Publ. Math. 68 (1988) 175-186.

[B-N] D. Bar-Natan, On the Vassiliev knot invariants, Topology 34 (1995) 423-472.

[BGRT] D. Bar-Natan, S. Garoufalidis, L. Rozansky and D. Thurston, The Aarhus integral of rational homology 3-spheres I-IV, preprints 1997.

[C] J. Conway, An enumeration of knots and links and some of their algebraic properties, Computational problems in abstract algebra, Pergamon Press, New-York 1970, 329-358.

[Da] A. Davidow, Casson's invariant and twisted double knots, Topology Appl. 58 (1994) 93-101.

$[\mathrm{GH}] \quad$ S. Garoufalidis, N. Habegger, The Alexander polynomial and finite type 3-manifold invariants, to appear in Math. Annalen.

[GO] S. Garoufalidis, T. Ohtsuki, On finite type 3-manifold invariants V: rational homology spheres, Proceedings of the Aarhus Conference, Geometry and Physics, Marcel Dekker (1996) 445-457.

[Ho] J. Hoste, The first coefficient of the Conway polynomial, Proc. Amer. Math. Soc. 95 (1985) 299302. 
[I] K. Ishibe, The Casson-Walker invariant for branched cyclic covers of $S^{3}$ branched over a doubled knot, Osaka J. Math. 34 (1997) 481-495.

[J] V. Jones, Hecke algebra representation of braid groups and link polynomials, Annals Math. 126 (1987) p. 335-388.

[Ka] L. Kauffman, On knots, Ann. of Math. Studies, 115, Princeton University Press, 1987.

[Ko] M. Kontsevich, Vassiliev's knot invariants, Adv. in Sov. Math., 16(2) (1993), 137-150.

[Ky] R.H. Kyle, Branched covering spaces and the quadratic form of links, Ann. of Math. 59 (1954) $539-548$,

[L] T.T.Q. Le, An invariant of integral homology 3-spheres which is universal for all finite type invariants, Soliton Geometry and Topology: on the crossroad, AMS Translations 2 Eds. V. Buchstaber, S. Novikov.

[LMMO] T.T.Q. Le, H. Murakami, J. Murakami, T. Ohtsuki, A three-manifold invariant derived from the universal Vassiliev-Kontsevich invariant, Proc. Japan Acad. Ser. A Math. Sci. 71 (1995) 125-127.

[Le] C. Lescop, Global surgery formula for the Casson-Walker invariant, Annals of Math Studies, 140 Princeton Univ. Press 1996.

[LMO] T.T.Q. Le, J. Murakami, T. Ohtsuki, A universal quantum invariant of 3-manifolds, Topology 37 (1998) 539-574.

[Li] A.S. Lipson, Link signature, Goeritz matrices and polynomial invariants, L' Enseign. Math. 36 (1990) 93-114.

[Mu] D. Mullins, The generalized Casson invariant for 2-fold branched covers of $S^{3}$ and the Jones polynomial, Topology, 32 (1993) 419-438.

[Oh] T. Ohtsuki, Finite type invariants of integral homology 3-spheres, J. Knot Theory and its Rami. 5 (1996) 101-115.

[RT1] N. Reshetikhin, V. Turaev, Ribbon graphs and their invariants derived from quantum groups, Commun. Math. Phys. 127 (1990) 1-26.

[RT2] N. Reshetikhin, V. Turaev, Invariants of 3-manifolds via link polynomials and quantum groups, Invent. Math. 103 (1991) 547-597.

[Wa] K. Walker, An extension of Casson's invariant, Ann. of Math. Studies 126, Princeton University Press, 1992.

[Wi] E.Witten, Quantum field theory and the Jones polynomial, Commun. Math. Physics. 121 (1989) 360-376.

Department of Mathematics, Harvard University, 1 Oxford Street, Cambridge, MA 02138, USA

E-mail address: stavros@math.harvard.edu 